\title{
Navier-Stokes equations with delays on unbounded domains
}

\author{
By María José Garrido-Atienza, \& Pedro Marín-Rubio † \\ Departamento de Ecuaciones Diferenciales y Análisis Numérico, \\ Universidad de Sevilla, \\ Apdo. de Correos 1160, \\ 41080-Sevilla. \\ Spain
}

Some results on the existence and uniqueness of solutions to Navier-Stokes equations when the domain is unbounded and the external force contains some hereditary characteristics are proved for both the evolutionary and the stationary cases. Exponential stability of the stationary solution is also established in dimension two.

Keywords: Navier-Stokes equations, variable and distributed delays, unbounded domains.

\section{Introduction and statement of the problem}

The Navier-Stokes equations govern the motion of usual fluids like water, air, oil, etc. These equations have been the object of numerous works since the first paper of Leray was published in 1933 (see Constantin \& Foias 1988; Lions 1969; Temam 1979, and the references therein), even with unbounded domains, allowing the possibility of channel and multi-channel flows among other variations (see for instance Rosa 1998; Temam 1979). On other hand, delay effects have been proved to be useful in many physical and biological situations. These situations may appear when we want to control the system (in a certain sense) by applying a force which takes into account not only the present state of the system but the history of the solution. To our knowledge, this has been rarely treated in the context of Navier-Stokes equations (cf. Caraballo \& Real 2001, 2003, 2004). To date, we have not found in the literature any work that considers the combination of delay terms and unbounded domains.

The aim of the paper is two-fold: firstly we consider several situations in which the external force contains some hereditary features and the domain is not bounded, and prove existence (for dimension $N=2$ and 3) and uniqueness $(N=2$ ) of solutions. In a second part of the paper the existence and uniqueness of a stationary solution are established in dimensions 2 and 3 , and, in the case $N=2$ exponential stability of the solution under an additional assumption.

Let $\Omega \subset \mathbb{R}^{N}$ ( $N=2$ or 3 ) be an open set with boundary $\Gamma$ that is not necessarily bounded but satisfies a Poincaré inequality:

There exists $\lambda_{1}>0$ such that $\int_{\Omega}|\phi|^{2} \mathrm{~d} x \leq \frac{1}{\lambda_{1}} \int_{\Omega}|\nabla \phi|^{2} \mathrm{~d} x, \quad \forall \phi \in H_{0}^{1}(\Omega)$.

$\dagger$ E-mails: mgarrido@us.es ; pmr@us.es 
Consider the following functional Navier-Stokes problem (for further details and notations see Lions 1969 and Temam 1979):

$$
\left\{\begin{array}{l}
\frac{\partial u}{\partial t}-\nu \Delta u+\sum_{i=1}^{N} u_{i} \frac{\partial u}{\partial x_{i}}=f(t)-\nabla p+g\left(t, u_{t}\right) \quad \text { in }(0, T) \times \Omega \\
\operatorname{div} u=0 \quad \text { in }(0, T) \times \Omega \\
u=0 \text { on }(0, T) \times \Gamma \\
u(0, x)=u^{0}(x), \quad x \in \Omega, \\
u(t, x)=\phi(t, x), t \in(-h, 0) \quad x \in \Omega
\end{array}\right.
$$

where we assume that $T>0$ is given, $\nu>0$ is the kinematic viscosity, $u$ is the velocity field of the fluid, $p$ the pressure, $u^{0}$ the initial velocity field, $f$ a nondelayed external force field, $g$ another external force containing some hereditary characteristic and $\phi$ the initial datum in the interval of time $(-h, 0)$, where $h$ is a positive fixed number.

To start, we consider the following usual abstract spaces:

$$
\mathcal{V}=\left\{u \in\left(C_{0}^{\infty}(\Omega)\right)^{N}: \operatorname{div} u=0\right\},
$$

$H=$ the closure of $\mathcal{V}$ in $\left(L^{2}(\Omega)\right)^{N}$ with the norm $|\cdot|$, and inner product $(\cdot, \cdot)$ where for $u, v \in\left(L^{2}(\Omega)\right)^{N}$,

$$
(u, v)=\sum_{j=1}^{N} \int_{\Omega} u_{j}(x) v_{j}(x) \mathrm{d} x,
$$

$V=$ the closure of $\mathcal{V}$ in $\left(H_{0}^{1}(\Omega)\right)^{N}$ with the norm [thanks to $\left.(1.1)\right]\|\cdot\|$ associated to the inner product $((\cdot, \cdot))$, where for $u, v \in\left(H_{0}^{1}(\Omega)\right)^{N}$,

$$
((u, v))=\sum_{i, j=1}^{N} \int_{\Omega} \frac{\partial u_{j}}{\partial x_{i}} \frac{\partial v_{j}}{\partial x_{i}} \mathrm{~d} x
$$

It follows that $V \subset H \equiv H^{\prime} \subset V^{\prime}$, where the injections are dense and continuous. We will use $\|\cdot\|_{*}$ for the norm in $V^{\prime}$ and $\langle\cdot, \cdot\rangle$ for the duality $\left\langle V^{\prime}, V\right\rangle$. Now we denote $a(u, v)=((u, v))$, and define the trilinear form $b$ on $V \times V \times V$ by

$$
b(u, v, w)=\sum_{i, j=1}^{N} \int_{\Omega} u_{i} \frac{\partial v_{j}}{\partial x_{i}} w_{j} \mathrm{~d} x \quad \forall u, v, w \in V .
$$

Let $X$ be a Banach space. Given a function $u:(-h, T) \rightarrow X$, for each $t \in(0, T)$ we denote by $u_{t}$ the function defined on $(-h, 0)$ by the relation $u_{t}(s)=u(t+s), s \in$ $(-h, 0)$.

In order to state the problem in the correct framework, let us first establish suitable assumptions on the term in which the delay is present.

In a general way, let $X$ and $Y$ be two separable Banach spaces, and $g:[0, T] \times$ $C^{0}([-h, 0] ; X) \rightarrow Y$ such that

(I) for all $\xi \in C^{0}([-h, 0] ; X)$, the mapping $t \in[0, T] \rightarrow g(t, \xi) \in Y$ is measurable, 
(II) for each $t \in[0, T], g(t, 0)=0$,

(III) there exists $L_{g}>0$ such that $\forall t \in[0, T], \forall \xi, \eta \in C^{0}([-h, 0] ; X)$

$$
\|g(t, \xi)-g(t, \eta)\|_{Y} \leq L_{g}\|\xi-\eta\|_{C^{0}([-h, 0] ; X)},
$$

(IV) there exists $C_{g}>0$ such that $\forall t \in[0, T], \forall u, v \in C^{0}([-h, T] ; X)$

$$
\int_{0}^{t}\left\|g\left(s, u_{s}\right)-g\left(s, v_{s}\right)\right\|_{Y}^{2} \mathrm{~d} s \leq C_{g} \int_{-h}^{t}\|u(s)-v(s)\|_{X}^{2} \mathrm{~d} s .
$$

Observe that (I)-(III) imply that given $u \in C^{0}([-h, T] ; X)$, the function $g_{u}: t \in$ $[0, T] \rightarrow Y$ defined by $g_{u}(t)=g\left(t, u_{t}\right) \forall t \in[0, T]$, is measurable (see Bensoussan et al. 1992) and, in fact, belongs to $L^{\infty}(0, T ; Y)$. Then, thanks to (IV), the mapping

$$
\mathcal{G}: u \in C^{0}([-h, T] ; X) \rightarrow g_{u} \in L^{2}(0, T ; Y)
$$

has a unique extension to a mapping $\widetilde{\mathcal{G}}$ which is uniformly continuous from $L^{2}(-h, T ; X)$ into $L^{2}(0, T ; Y)$. From now on, we will denote $g\left(t, u_{t}\right)=\widetilde{\mathcal{G}}(u)(t)$ for each $u \in$ $L^{2}(-h, T ; X)$, and thus, $\forall t \in[0, T], \forall u, v \in L^{2}(-h, T ; X)$, we will have

$$
\int_{0}^{t}\left\|g\left(s, u_{s}\right)-g\left(s, v_{s}\right)\right\|_{Y}^{2} \mathrm{~d} s \leq C_{g} \int_{-h}^{t}\|u(s)-v(s)\|_{X}^{2} \mathrm{~d} s .
$$

With the convention above, assume that $u^{0} \in H, \phi \in L^{2}(-h, 0 ; V), f \in L^{2}\left(0, T ; V^{\prime}\right)$, and the following delay operators:

$$
g_{1}:[0, T] \times C^{0}([-h, 0] ; V) \rightarrow\left(L^{2}(\Omega)\right)^{N}
$$

satisfying hypotheses (I)-(IV) with $X=V, Y=\left(L^{2}(\Omega)\right)^{N}, L_{g_{1}}=L_{1}$ and $C_{g_{1}}=C_{1}$, and

$$
g_{2}:[0, T] \times C^{0}([-h, 0] ; V) \rightarrow V^{\prime}
$$

satisfying hypotheses (I)-(IV) with $X=V, Y=V^{\prime}, L_{g_{2}}=L_{2}$ and $C_{g_{2}}=C_{2}$.

We are interested in the following problem:

$$
\left\{\begin{array}{l}
\text { To find } u \in L^{2}(-h, T ; V) \cap L^{\infty}(0, T ; H) \text { such that, for all } v \in V, \\
\begin{array}{cc}
\frac{\mathrm{d}}{\mathrm{d} t}(u(t), v)+\nu a(u(t), v)+b(u(t), u(t), v)=\langle f(t), v\rangle+\left(g_{1}\left(t, u_{t}\right), v\right) \\
u(0)=u^{0}, u(t)=\phi(t), \quad t \in(-h, 0), & +\left\langle g_{2}\left(t, u_{t}\right), v\right\rangle,
\end{array}
\end{array}\right.
$$

where the equation in (1.2) must be understood in the sense of $\mathcal{D}^{\prime}(0, T)$.

Remark 1.1. Observe that the terms in (1.2) are well defined. In particular, by hypotheses (I)-(IV), if $u \in L^{2}(-h, T ; V)$ the term $g_{1}\left(t, u_{t}\right)$ defines a function in $L^{2}\left(0, T ;\left(L^{2}(\Omega)\right)^{N}\right)$, and the term $g_{2}\left(t, u_{t}\right)$ defines a function in $L^{2}\left(0, T ; V^{\prime}\right)$. Thus (see Lions 1969), if $u \in L^{2}(-h, T ; V) \cap L^{\infty}(0, T ; H)$ satisfies the equation in $(1.2), u$ is weakly continuous from $[0, T]$ into $H$, and therefore the initial condition $u(0)=u^{0}$ makes sense. Of course, for $N=2$, if there exists a solution $u$ to the problem (1.2), it then belongs to the space $C^{0}([0, T] ; H)$.

In Section 2 we shall prove existence of solutions to (1.2) and the uniqueness of solution to the problem in the case $N=2$. In Section 3, general situations containing delayed terms -variable and distributed- are considered. We finish with the study of existence and uniqueness of a stationary solution and its exponential stability (for $N=2$ ) in Section 4 . 


\section{Existence of solutions}

In this section we will prove a general theorem on the existence of solutions when $N=2$ or 3 , and uniqueness if $N=2$.

In the proof of existence we will need the following two results:

Theorem 2.1. (cf. [Caraballo \& Real 2001, Theorem A.1]) Let $u^{0} \in \mathbb{R}^{m}, \phi \in$ $L^{2}\left(-h, 0 ; \mathbb{R}^{m}\right), k \in L^{2}\left(0, T ; \mathbb{R}^{m}\right), g:[0, T] \times C^{0}\left([-h, 0] ; \mathbb{R}^{m}\right) \rightarrow \mathbb{R}^{m}$ satisfying hypotheses (I)-(IV) with $X=Y=\mathbb{R}^{m}$, and $f:[0, T] \times \mathbb{R}^{m} \rightarrow \mathbb{R}^{m}$ a continuous function such that $f(t, 0)=0$ and for all $n>0$ there exists $L_{n}>0$ such that

$$
|f(t, u)-f(t, v)|_{\mathbb{R}^{m}} \leq L_{n}|u-v|_{\mathbb{R}^{m}}, \quad \forall|u|_{\mathbb{R}^{m}} \leq n,|v|_{\mathbb{R}^{m}} \leq n, \quad \forall t \in[0, T] .
$$

Then:

a) For each $t_{*} \in(0, T]$ there exists at most one solution to the problem

$$
\left\{\begin{array}{l}
\text { To find } u \in L^{2}\left(-h, t_{*} ; \mathbb{R}^{m}\right) \cap C^{0}\left(\left[0, t_{*}\right] ; \mathbb{R}^{m}\right) \text { such that } \\
u(t)=\phi(t), \quad t \in(-h, 0), \\
u(t)=u^{0}+\int_{0}^{t} f(s, u(s)) \mathrm{d} s+\int_{0}^{t} g\left(s, u_{s}\right) \mathrm{d} s+\int_{0}^{t} k(s) \mathrm{d} s \quad \forall t \in\left[0, t_{*}\right] .
\end{array}\right.
$$

b) There exists $t_{*} \in(0, T]$ such that there exists one (and only one) solution to the problem (2.1).

c) Suppose that there exists a constant $C>0$ such that if $t_{*} \in(0, T]$ is such that there is a solution $u$ of $(2.1)$, then $\max _{t \in\left[0, t_{*}\right]} \mid u(t){\mid \mathbb{R}^{m}} \leq C$. Then, under this additional assumption, there exists a solution to problem (2.1) with $t_{*}=T$.

Theorem 2.2. (cf. [Simon 2003, Corollary 2.34]) Let $\Theta$ be a bounded open set of $\mathbb{R}^{d}$, and $X \subset E$ Banach spaces with compact injection. Consider $1 \leq r<q \leq \infty$. Suppose $F \subset L^{r}(\Theta ; E)$ satisfies

(i) $\forall \omega \subset \subset \Theta, \sup _{f \in F}\left\|\tau_{h} f-f\right\|_{L^{r}(\omega ; E)} \rightarrow 0$ when $h \rightarrow 0$ [where $\tau_{h} f$ is the translation: $\left.\left(\tau_{h} f\right)(x)=f(x+h)\right]$,

(ii) $F$ is bounded in $L^{q}(\Theta ; E) \cap L^{1}(\Theta ; X)$.

Then $F$ is precompact in $L^{r}(\Theta ; E)$.

The main result in this section needs extra notation for an additional condition $(\mathrm{V})$, which will be discussed in some detail below in Remark 2.5.

Denote $\mathcal{V}(\mathcal{O})$ the same space as $\mathcal{V}$ but with an open set $\mathcal{O}$ instead of $\Omega$, and analogously define $V(\mathcal{O})$ the closure of $\mathcal{V}(\mathcal{O})$ in $\left(H_{0}^{1}(\Omega)\right)^{N}$.

Theorem 2.3. Let $u^{0} \in H, \phi \in L^{2}(-h, 0 ; V), f \in L^{2}\left(0, T ; V^{\prime}\right)$, and assume that $g_{1}:[0, T] \times C^{0}([-h, 0] ; V) \rightarrow\left(L^{2}(\Omega)\right)^{N}$ and $g_{2}:[0, T] \times C^{0}([-h, 0] ; V) \rightarrow V^{\prime}$ satisfy hypotheses (I)-(IV) in their corresponding spaces. Then:

a) If $N=2$ and $\nu^{2}>C_{2}$, there exists at most one solution to problem (1.2).

b) If $N \in\{2,3\}$ and $\nu^{2}>C_{2}$, there exists a solution to (1.2) if, in addition, the following assumption $(\mathrm{V})$ holds:

(V) If $v^{m}$ converges weakly to $v$ in $L^{2}(-h, T ; V)$, weakly-star in $L^{\infty}(0, T ; H)$, and strongly in $L^{2}\left(-h, T ;\left(L^{2}(\mathcal{O})\right)^{N}\right)$ for a bounded open set $\mathcal{O} \subset \Omega$ with smooth boundary, then $g_{i}\left(\cdot, v^{m}\right)$ converges weakly to $g_{i}(\cdot, v$. $)$ in $L^{2}\left(0, T ; V(\mathcal{O})^{\prime}\right)$ for $i=1,2$. 
Proof. a) Uniqueness for $N=2$ follows as the case with bounded domain given in Theorem 2.1 in [Caraballo \& Real 2001] and it is reproduced here for the sake of completeness. If $\nu^{2}>C_{2}$, let $u, v$ be two solutions to (1.2) and set $w=u-v$. Then, from the energy equality, and the bound for the trilinear form (see Ladyzhenskaya 1992), it follows that for all $t \in(0, T)$

$$
\begin{aligned}
|w(t)|^{2}+2 \nu \int_{0}^{t}\|w(s)\|^{2} \mathrm{~d} s= & -2 \int_{0}^{t} b(w(s), u(s), w(s)) \mathrm{d} s \\
& +2 \int_{0}^{t}\left(g_{1}\left(s, u_{s}\right)-g_{1}\left(s, v_{s}\right), w(s)\right) \mathrm{d} s \\
& +2 \int_{0}^{t}\left\langle g_{2}\left(s, u_{s}\right)-g_{2}\left(s, v_{s}\right), w(s)\right\rangle \mathrm{d} s \\
\leq & 2^{1 / 2} \int_{0}^{t}|w(s)|\|w(s)\|\|u(s)\| \mathrm{d} s \\
& +2 \int_{0}^{t}\left|g_{1}\left(s, u_{s}\right)-g_{1}\left(s, v_{s}\right)\right||w(s)| \mathrm{d} s \\
& +2 \int_{0}^{t}\left\|g_{2}\left(s, u_{s}\right)-g_{2}\left(s, v_{s}\right)\right\|_{*}\|w(s)\| \mathrm{d} s .
\end{aligned}
$$

Then, from assumption (IV), taking into account that $w(s)=0$ for $s \in(-h, 0)$, and denoting $2 \varepsilon=\nu-\sqrt{C_{2}}>0$, we have for all $t \in(0, T)$

$$
\begin{aligned}
|w(t)|^{2}+2 \nu \int_{0}^{t}\|w(s)\|^{2} \mathrm{~d} s \leq & \frac{1}{2 \varepsilon} \int_{0}^{t}|w(s)|^{2}\|u(s)\|^{2} \mathrm{~d} s+\varepsilon \int_{0}^{t}\|w(s)\|^{2} \mathrm{~d} s \\
& +\frac{C_{1}}{\varepsilon} \int_{0}^{t}|w(s)|^{2} \mathrm{~d} s+\varepsilon \int_{0}^{t}\|w(s)\|^{2} \mathrm{~d} s \\
& +2 \sqrt{C_{2}} \int_{0}^{t}\|w(s)\|^{2} \mathrm{~d} s
\end{aligned}
$$

and so,

$$
|w(t)|^{2}+2 \varepsilon \int_{0}^{t}\|w(s)\|^{2} \mathrm{~d} s \leq \frac{1}{2 \varepsilon} \int_{0}^{t}|w(s)|^{2}\|u(s)\|^{2} \mathrm{~d} s+\frac{C_{1}}{\varepsilon} \int_{0}^{t}|w(s)|^{2} \mathrm{~d} s
$$

from which uniqueness follows thanks to the Gronwall lemma: indeed, denoting $C=\max \left(2^{-1}, C_{1}\right) / \varepsilon$, we have that

$$
\frac{\mathrm{d}}{\mathrm{d} t}\left(|w(t)|^{2} \exp \left\{-C \int_{0}^{t}\left(\|u(s)\|^{2}+1\right) \mathrm{d} s\right\}\right) \leq 0 .
$$

b) Now for the existence, we assume $N \in\{2,3\}, \nu^{2}>C_{2}$ and that condition (V) holds.

We will develop a proof based on the unbounded case without delays (see for instance Temam 1979), and on the case with delays on bounded domains (Caraballo \& Real 2001), but with both difficulties treated jointly. 
Consider an orthonormal basis $B=\left\{w_{1}, \ldots, w_{n}, \ldots\right\} \subset \mathcal{V}$ of $H$ such that linear combinations of elements of $B$ are dense in $V . \dagger$ [Notice one usually takes a special basis, using the Stokes operator. This is not valid here since compactness is lost, and it will have influence on the way one can obtain a convergent subsequence, because standard estimates on the derivatives of the velocity fields are not valid neither.]

Let us denote $V_{m}=\operatorname{span}\left[w_{1}, \ldots, w_{m}\right], P_{V_{m}}^{H}: H \rightarrow V_{m}$ the projector given by $P_{V_{m}}^{H} u=\sum_{j=1}^{m}\left(u, w_{j}\right) w_{j}$. We will also denote $P_{V_{m}}^{V}: V \rightarrow V_{m}$ the projector given by $P_{V_{m}}^{V} u=\sum_{j=1}^{m}\left(\left(v, \tilde{w}_{j}\right)\right) \tilde{w}_{j}$ (where the sequence $\left\{\tilde{w}_{1}, \ldots, \tilde{w}_{n}\right\}$ comes from the Gram-Schmidt orthonormalization process in $V$; this will be useful since the initial convergence in $(-h, 0)$ must hold in $V)$.

Finally, define $u^{m}(t)=\sum_{j=1}^{m} \gamma_{m j}(t) w_{j}$, where

$$
\left\{\begin{array}{l}
u^{m} \in L^{2}\left(-h, T ; V_{m}\right) \cap C^{0}\left([0, T] ; V_{m}\right) \\
\frac{\mathrm{d}}{\mathrm{d} t}\left(u^{m}(t), w_{j}\right)+\nu a\left(u^{m}(t), w_{j}\right)+b\left(u^{m}(t), u^{m}(t), w_{j}\right)=\left\langle f(t), w_{j}\right\rangle+ \\
\quad+\left(g_{1}\left(t, u_{t}^{m}\right), w_{j}\right)+\left\langle g_{2}\left(t, u_{t}^{m}\right), w_{j}\right\rangle \quad \text { in } \mathcal{D}^{\prime}(0, T), \quad 1 \leq j \leq m, \\
u^{m}(0)=P_{V_{m}}^{H} u^{0}, u^{m}(t)=P_{V_{m}}^{V} \phi(t), \quad t \in(-h, 0) .
\end{array}\right.
$$

The preceding is a system of ordinary functional differential equations in the unknown $\gamma^{m}(t)=\left(\gamma_{m 1}(t), \ldots, \gamma_{m m}(t)\right)$. Existence and uniqueness of solution is obtained by applying Theorem 2.1 stated above.

Observe that problem $(2.2)$ has one solution defined in an interval $\left[0, t_{*}\right]$ with $0<t_{*} \leq T$. However, as usual, it can be deduced by the a priori estimates below, we can set $t_{*}=T$.

In fact, multiplying in $(2.2)$ by $\gamma_{m j}(t)$ and summing in $j$, we get for all $t \in\left[0, t_{*}\right]$

$$
\begin{aligned}
\left|u^{m}(t)\right|^{2}+2 \nu \int_{0}^{t}\left\|u^{m}(s)\right\|^{2} \mathrm{~d} s \leq & \left|u^{0}\right|^{2}+2 \int_{0}^{t}\left\langle f(s), u^{m}(s)\right\rangle \mathrm{d} s \\
& +2 \int_{0}^{t}\left(g_{1}\left(s, u_{s}^{m}\right), u^{m}(s)\right) \mathrm{d} s \\
& +2 \int_{0}^{t}\left\langle g_{2}\left(s, u_{s}^{m}\right), u^{m}(s)\right\rangle \mathrm{d} s
\end{aligned}
$$

and arguing in a similar manner as in the proof of uniqueness in the 2-dimensional case, we easily get two constants (depending on $\phi, \nu, f, g_{1}, g_{2}, h, T$, but not on $m$ nor $\left.t_{*}\right) K_{1}$ and $K_{2}$ such that

$$
\sup _{t \in\left[0, t_{*}\right]}\left|u^{m}(t)\right|^{2} \leq K_{1}, \quad \int_{0}^{t_{*}}\left\|u^{m}(s)\right\|^{2} \mathrm{~d} s \leq K_{2} .
$$

So we can take $t_{*}=T$, and obtain that $\left\{u^{m}\right\}$ is bounded in $L^{2}(0, T ; V) \cap L^{\infty}(0, T ; H)$, so there exists a subsequence, relabelled the same, such that

$$
u^{m} \rightarrow u \text { in } L^{2}(0, T ; V) \text { weakly and in } L^{\infty}(0, T ; H) \text { weak-star as } m \rightarrow \infty .
$$

† This can be obtained as follows: $V$ is separable (since it is a subset of $\left(H_{0}^{1}(\Omega)\right)^{N}$ ), and by definition $\mathcal{V}$ is dense in $V$ and $H$, which implies that $V$ is also dense in $H$. Thus, given a sequence $\left\{v_{i}\right\}_{i>1} \subset V$ dense in $V$, we may take a sequence $\left\{w_{n}^{i}\right\}_{i, n \geq 1} \subset \mathcal{V}$ which accumulates to every point $v_{i}$, and therefore, linear combinations of these elements are dense in $V$ and $H$. Since $V \subset H$ are vectorial subspaces of $\left(L^{2}(\Omega)\right)^{N}$, linear (in)dependence is equivalent considered in any of them, whence $B$ is obtained applying the Gram-Schmidt orthonormalization process. 
Moreover, observe that $u^{m}=P_{V_{m}}^{V} \phi$ in $(-h, 0)$ converges to $\phi$ in $L^{2}(-h, 0 ; V)$, and, in particular, thanks to (IV), $g_{1}\left(\cdot, u^{m}\right)+g_{2}\left(\cdot, u^{m}\right)$ is bounded in $L^{2}\left(0, T ; V^{\prime}\right)$.

As we noticed before, when it is possible to choose a special basis (in bounded domains it is so), standard estimates on $\left\|\frac{\mathrm{d}}{\mathrm{d} t} u^{m}\right\|_{L^{2}\left(0, T ; V^{\prime}\right)}\left(\right.$ for $N=2$, and $L^{4 / 3}\left(0, T ; V^{\prime}\right)$ for $N=3$ ) allow us to obtain a compactness result: a subsequence $u^{m}$ converges to $u$ in $L^{2}(0, T ; H)$.

Here we will have a similar result but not in a straightforward way, nor on the whole domain $\Omega$. Actually, what holds in this case is the following:

For any bounded open set $\mathcal{O} \subset \Omega$ there exists a subsequence (depending on $\mathcal{O}$ which we relabel) satisfying

$$
\left.\left.u^{m}\right|_{\mathcal{O}} \rightarrow u\right|_{\mathcal{O}} \text { in } L^{2}\left(0, T ;\left(L^{2}(\mathcal{O})\right)^{N}\right)
$$

For the sake of clarity, we postpone the proof (we will use Theorem 2.2) to Lemma 2.4 below.

Now, let $\psi$ be a continuously differentiable function on $[0, T]$ with $\psi(T)=$ 0 . Consider equation $(2.2)$ and a fixed element $w_{j}$ of $B$. Since $\left(u^{m}(\cdot), w_{j}\right) \psi(\cdot) \in$ $W^{1,1}(0, T)$ (actually in $H^{1}(0, T)$ for $N=2$, and $W^{1,4 / 3}(0, T)$ for $N=3$ ) we have

$$
\begin{gathered}
-\int_{0}^{T}\left(u^{m}(t), \psi^{\prime}(t) w_{j}\right) \mathrm{d} t+\nu \int_{0}^{T}\left(\left(u^{m}(t), w_{j} \psi(t)\right)\right) \mathrm{d} t \\
+\int_{0}^{T} b\left(u^{m}(t), u^{m}(t), w_{j} \psi(t)\right) \mathrm{d} t=\left(u^{m}(0), w_{j}\right) \psi(0)+\int_{0}^{T}\left\langle f(t), w_{j} \psi(t)\right\rangle \mathrm{d} t \\
+\int_{0}^{T}\left(g_{1}\left(t, u_{t}^{m}\right), w_{j} \psi(t)\right) \mathrm{d} t+\int_{0}^{T}\left\langle g_{2}\left(t, u_{t}^{m}\right), w_{j} \psi(t)\right\rangle \mathrm{d} t .
\end{gathered}
$$

Taking a diagonal subsequence, denoted again $u^{m}$, that satisfies (2.5) for a sequence of regular bounded open sets $\mathcal{O}_{j} \subset \Omega$ that contain all supports of functions $w_{j}$ of the basis, we may now pass to the limit, thanks to the weak convergence in (2.4) and condition (V) too. Thus, we obtain (first for any $w \in\left\{w_{1}, w_{2}, \ldots\right\}$, and by density for every $w \in V)$ :

$$
\begin{gathered}
-\int_{0}^{T}\left(u(t), \psi^{\prime}(t) w\right) \mathrm{d} t+\nu \int_{0}^{T}((u(t), w \psi(t))) \mathrm{d} t \\
+\int_{0}^{T} b(u(t), u(t), w \psi(t)) \mathrm{d} t=\left(u^{0}, w\right) \psi(0)+\int_{0}^{T}\langle f(t), w \psi(t)\rangle \mathrm{d} t \\
+\int_{0}^{T}\left(g_{1}\left(t, u_{t}\right), w \psi(t)\right) \mathrm{d} t+\int_{0}^{T}\left\langle g_{2}\left(t, u_{t}\right), w \psi(t)\right\rangle \mathrm{d} t .
\end{gathered}
$$

Writing (2.6) for $\psi \in \mathcal{D}(0, T), u$ satisfies (2.2) in the distribution sense.

By Remark 1.1 it makes sense to wonder about the value at time $t=0$. Now, since $\left(u(t), w_{j}\right) \psi(t) \in W^{1,4 / 3}(0, T)$, for both $N=2$ or 3 , arguing as before, we obtain an analogous expression to $(2.6)$ with $(u(0), w)$ instead of $\left(u^{0}, w\right)$. This implies $\left(u(0)-u^{0}, w\right)=0$ for all $w \in V$, so $u(0)=u^{0}$. 
For the following result, let us observe that the constants appearing in standard estimates of the trilinear form $b$-for a proof see for instance Temam 1979, lemmas 3.3, 3.4, 3.5 and Theorem 3.3, pp. 291 and forward- may be improved (cf. Ladyzhenskaya 1992 for the case $N=2$ ). Although it is not essential to make the most of them for existence and uniqueness, since we will establish a stability result later, we use them in order to ensure it under the minimal conditions.

Lemma 2.4. Under the assumptions of Theorem 2.3, the sequence $u^{m}$ given in (2.2) is precompact in the following sense: suppose a bounded open set $\mathcal{O} \subset \Omega$ is given, then there exists a subsequence depending on $\mathcal{O}$, which we relabel, such that

$$
\left.\left.u^{m}\right|_{\mathcal{O}} \rightarrow u\right|_{\mathcal{O}} \text { in } L^{2}\left(0, T ;\left(L^{2}(\mathcal{O})\right)^{N}\right)
$$

where $u$ is the weak limit given in (2.4).

Proof. We will adapt the proof of Theorem 9.4 [cf. Simon 2003] to check our situation fits to the Theorem 2.2. More exactly, we claim it can be applied taking $r=2$, $q=+\infty, \Theta=(0, T)$.

For the set $\mathcal{O} \subset \Omega$ let us make precise a technical detail: if $\mathcal{O} \subset \subset \Omega$ one may obtain a finite recovering of balls, denoted $\tilde{\mathcal{O}} \subset \Omega$, which is bounded and open, and then $X=\left(H^{1}(\tilde{\mathcal{O}})\right)^{N} \subset E=\left(L^{2}(\tilde{\mathcal{O}})\right)^{N}$ with compact injection.

However, for a general $\mathcal{O} \subset \Omega$ the above comment may not be true since $\mathcal{O}$ and $\Omega$ can share part of their boundaries. The compact injection from $H^{1}$ may not hold for lack of regularity on the boundary (it is not imposed for $\Gamma$ ), however it does in $H_{0}^{1}$. One may then use a truncation argument (see for instance Rosa 1998): fix $\chi \in C^{1}\left(\mathbb{R}_{+}\right)$with $\chi(s)=1$ for $s \in[0,1]$ and $\chi(s)=0$ for $s \geq 4$. Consider $\mathcal{O}$ as in the statement, let $R>0$ be such that $\mathcal{O} \subset B(0, R)$ and denote $\tilde{\mathcal{O}}=\Omega \cap B(0,2 R)$, and $u^{m, R}(x)=u^{m}(x) \chi\left(|x|^{2} / R^{2}\right)$. Again the compactness holds for $X=\left(H_{0}^{1}(\tilde{\mathcal{O}})\right)^{N} \subset E=\left(L^{2}(\tilde{\mathcal{O}})\right)^{N}$ with compact injection, and we conserve the original functions $u^{m}$ on $\Omega \cap B(0, R)$.

For the sake of clarity, we continue the proof directly with $u^{m}$ instead of $u^{m, R}$. Since condition (ii) in Theorem 2.2 is obviously satisfied by (2.3), we concentrate on (i). Actually, we will prove that for the whole domain $\Omega$ the following property holds:

$$
\sup _{m \in \mathbb{N}}\left\|\tau_{h} u^{m}-u^{m}\right\|_{L^{2}\left(0, T-h ;\left(L^{2}(\Omega)\right)^{N}\right)} \rightarrow 0 \text { when } h \rightarrow 0 .
$$

Consider $h>0$ arbitrarily small. From (2.2) we deduce for $(t, t+h) \subset(0, T)$ that

$$
\begin{gathered}
\int_{\Omega}\left(u^{m}(t+h)-u(t)\right) w_{j} \mathrm{~d} x+\nu \int_{t}^{t+h} \int_{\Omega} \nabla u^{m}(s) \cdot \nabla w_{j} \mathrm{~d} x \mathrm{~d} s+\int_{t}^{t+h} b\left(u^{m}(s), u^{m}(s), w_{j}\right) \mathrm{d} s \\
=\int_{t}^{t+h}\left\langle f(s), w_{j}\right\rangle \mathrm{d} s+\int_{t}^{t+h} \int_{\Omega} g_{1}\left(s, u_{s}^{m}\right) w_{j} \mathrm{~d} x \mathrm{~d} s+\int_{t}^{t+h}\left\langle g_{2}\left(s, u_{s}^{m}\right), w_{j}\right\rangle \mathrm{d} s .
\end{gathered}
$$

Article submitted to Nonlinear Analysis TMA 
Multiplying by $\gamma_{m j}(t+h)-\gamma_{m j}(t)$ and summing in $j$ we obtain

$$
\begin{aligned}
\int_{\Omega}\left|u^{m}(t+h)-u(t)\right|^{2} \mathrm{~d} x= & -\nu \int_{t}^{t+h} \int_{\Omega} \nabla u^{m}(s) \cdot\left(\nabla u^{m}(t+h)-\nabla u^{m}(t)\right) \mathrm{d} x \mathrm{~d} s \\
& +\int_{t}^{t+h} b\left(u^{m}(s), u^{m}(s), u^{m}(t+h)-u^{m}(t)\right) \mathrm{d} s \\
& +\int_{t}^{t+h} \int_{\Omega} g_{1}\left(s, u_{s}^{m}\right)\left(u^{m}(t+h)-u^{m}(t)\right) \mathrm{d} x \mathrm{~d} s \\
& +\int_{t}^{t+h}\left\langle f(s)+g_{2}\left(s, u_{s}^{m}\right), u^{m}(t+h)-u^{m}(t)\right\rangle \mathrm{d} s
\end{aligned}
$$

The right hand side may be bounded by

$$
\begin{aligned}
& \nu\left|\nabla u^{m}(t+h)-\nabla u^{m}(t)\right| \int_{t}^{t+h}\left|\nabla u^{m}(s)\right| \mathrm{d} s \\
+ & \int_{t}^{t+h} G_{N}\left(\left|u^{m}(s)\right|,\left\|u^{m}(s)\right\|,\left\|u^{m}(t+h)-u^{m}(t)\right\|\right) \mathrm{d} s \\
+ & \int_{t}^{t+h}\left|g_{1}\left(s, u_{s}^{m}\right) \| u^{m}(t+h)-u^{m}(t)\right| \mathrm{d} s \\
+ & \int_{t}^{t+h}\left(\|f(s)\|_{*}+\left\|g_{2}\left(s, u_{s}^{m}\right)\right\|_{*}\right)\left\|u^{m}(t+h)-u^{m}(t)\right\| \mathrm{d} s
\end{aligned}
$$

where the trilinear form $b$ is bounded (depending on the dimension) by the function $G_{N}: \mathbb{R}^{3} \rightarrow \mathbb{R}$ defined as

$$
G_{N}(x, y, z)= \begin{cases}2^{-1 / 2} x y z & \text { if } N=2, \\ 2^{-1} x^{1 / 2} y^{3 / 2} z & \text { if } N=3 .\end{cases}
$$

Thus, using (1.1) and (2.3), we have proved that

$$
\int_{\Omega}\left|u^{m}(t+h)-u^{m}(t)\right|^{2} \mathrm{~d} x \leq\left\|u^{m}(t+h)-u^{m}(t)\right\| \int_{t}^{t+h} G_{m}(s) \mathrm{d} s
$$

where the function $G_{m}: \mathbb{R} \rightarrow \mathbb{R}$ is defined (recall definition given in (2.7)) as

$G_{m}(s)= \begin{cases}\nu\left\|u^{m}(s)\right\|+\left(2^{-1} K_{1}\right)^{1 / 2}\left\|u^{m}(s)\right\|+\|f(s)\|_{*}+\left\|g_{2}\left(s, u_{s}^{m}\right)\right\|_{*}+\lambda_{1}^{-1 / 2}\left|g_{1}\left(s, u_{s}^{m}\right)\right| & \text { if } N=2, \\ \nu\left\|u^{m}(s)\right\|+2^{-1} K_{1}^{1 / 4}\left\|u^{m}(s)\right\|^{3 / 2}+\|f(s)\|_{*}+\left\|g_{2}\left(s, u_{s}^{m}\right)\right\|_{*}+\lambda_{1}^{-1 / 2}\left|g_{1}\left(s, u_{s}^{m}\right)\right| & \text { if } N=3 .\end{cases}$

To finish the proof, we will estimate

$$
\begin{aligned}
\left\|\tau_{h} u^{m}-u^{m}\right\|_{L^{2}\left(0, T-h ;\left(L^{2}(\Omega)\right)^{N}\right)}^{2} & =\int_{0}^{T-h} \int_{\Omega}\left|\tau_{h} u^{m}-u^{m}\right|^{2} \mathrm{~d} x \mathrm{~d} t \\
& \leq \int_{0}^{T-h}\left\|u^{m}(t+h)-u^{m}(t)\right\| \int_{t}^{t+h} G_{m}(s) \mathrm{d} s \mathrm{~d} t .
\end{aligned}
$$

For the right hand side, the Fubini theorem yields, using the function

$$
\bar{s}= \begin{cases}0 & \text { if } \quad s \leq 0 \\ s & \text { if } \quad 0<s \leq T-h \\ T-h & \text { if } \quad s>T-h\end{cases}
$$


to

$$
\begin{aligned}
\int_{0}^{T-h}\left\|u^{m}(t+h)-u^{m}(t)\right\| \int_{t}^{t+h} G_{m}(s) \mathrm{d} s \mathrm{~d} t & \leq \int_{0}^{T} G_{m}(s) \int_{\overline{s-h}}^{\bar{s}}\left\|u^{m}(t+h)-u^{m}(t)\right\| \mathrm{d} t \mathrm{~d} s \\
& \leq 2\left(h K_{2}\right)^{1 / 2} \int_{0}^{T} G_{m}(s) \mathrm{d} s
\end{aligned}
$$

where we have used Young inequality, (2.3) and the fact that $0 \leq \bar{s}-\overline{s-h} \leq h$ for $\int \frac{\bar{s}}{s-h}\left\|u^{m}(t+h)-u^{m}(t)\right\| \mathrm{d} t$. Indeed,

$$
\begin{aligned}
\int_{\overline{s-h}}^{\bar{s}}\left\|u^{m}(t+h)-u^{m}(t)\right\| \mathrm{d} t & \leq\left(\int_{\overline{s-h}}^{\bar{s}} \mathrm{~d} t\right)^{1 / 2}\left(\int_{\overline{s-h}}^{\bar{s}}\left\|u^{m}(t+h)-u^{m}(t)\right\|^{2} \mathrm{~d} t\right)^{1 / 2} \\
& \leq 2 h^{1 / 2}\left(\int_{0}^{T-h} \int_{\Omega}\left|\nabla u^{m}\right|^{2} \mathrm{~d} x \mathrm{~d} t\right)^{1 / 2} \leq 2 h^{1 / 2} K_{2}^{1 / 2}
\end{aligned}
$$

To conclude, we observe that $\int_{0}^{T} G_{m}(s) \mathrm{d} s$ is bounded, for $N=2$ or 3 . For instance, suppose $N=2$, then one has

$$
\begin{aligned}
& \int_{0}^{T}\left(\left(\nu+\left(2^{-1} K_{1}\right)^{1 / 2}\right)\left\|u^{m}(s)\right\|+\|f(s)\|_{*}+\left\|g_{2}\left(s, u_{s}^{m}\right)\right\|_{*}+\lambda_{1}^{-1 / 2}\left|g_{1}\left(s, u_{s}^{m}\right)\right|\right) \\
\leq & \left(\nu+\left(2^{-1} K_{1}\right)^{1 / 2}\right) \sqrt{T}\left(\int_{0}^{T}\left\|u^{m}(s)\right\|^{2} \mathrm{~d} s\right)^{1 / 2}+\sqrt{T}\left(\int_{0}^{T}\|f(s)\|_{*}^{2} \mathrm{~d} s\right)^{1 / 2} \\
& +\sqrt{T}\left(\int_{0}^{T}\left\|g_{2}\left(s, u_{s}^{m}\right)\right\|_{*}^{2} \mathrm{~d} s\right)^{1 / 2}+\sqrt{T} \lambda_{1}^{-1 / 2}\left(\int_{0}^{T}\left|g_{1}\left(s, u_{s}^{m}\right)\right|^{2} \mathrm{~d} s\right)^{1 / 2},
\end{aligned}
$$

and assumptions (II) and (IV) give bounds for the integrals of $g_{i}$ in terms of the bounds in (2.3). The case $N=3$ is analogous, since the only difference, namely $\int_{0}^{T}\left\|u^{m}(s)\right\|^{3 / 2} \mathrm{~d} s$, is also bounded by $T^{1 / 4}\left(\int_{0}^{T}\left\|u^{m}(s)\right\|^{2} \mathrm{~d} s\right)^{3 / 4}$.

Remark 2.5. On condition (V)

(i) The reason we formulate condition (V) with so many assumptions is to state it in the weakest way, since all these hypothesis are satisfied by $\left\{u^{m}\right\}$.

(ii) By the assumptions on $\left\{v^{m}\right\}$ and (IV) we already know $\left\{g_{i}\left(\cdot, v^{m}\right)\right\}, i=1,2$ are weakly relatively compact in $L^{2}\left(0, T ;\left(L^{2}(\Omega)\right)^{N}\right)$ and $L^{2}\left(0, T ; V^{\prime}\right)$ respectively, and so both in $L^{2}\left(0, T ; V(\mathcal{O})^{\prime}\right)$ for any $\mathcal{O} \subset \Omega$. What claimed is that if $\mathcal{O} \subset \Omega$ is bounded and has a smooth boundary, the weak limit is precisely $g_{i}(\cdot, v$. $)$.

When Condition (V) is satisfied in all "good" $\mathcal{O} \subset \Omega$ (actually, it is enough for all $\left.\mathcal{O}_{j} \supset \operatorname{supp}\left(w_{j}\right)\right)$, then the convergence holds in $L^{2}\left(0, T ; V^{\prime}\right)$. Indeed, consider $\varphi \in$ $V$. We check that $\lim _{m}\left\langle g_{i}\left(\cdot, v^{m}\right), \varphi\right\rangle=\left\langle g_{i}(\cdot, v\right.$. $\left.), \varphi\right\rangle$. Take a sequence $\varphi_{n} \in \mathcal{V}$ such that $\varphi_{n} \rightarrow \varphi$ in $V$, and fix $\varepsilon>0$. Consider $n_{\varepsilon}$ such that $\max \left\{\left\|g_{i}\left(\cdot, v_{\cdot}^{m}\right)\right\|, \| g_{i}(\cdot, v\right.$. $\left.)\right\} \| \varphi_{n}-$ $\varphi \| \leq \varepsilon / 2$ for all $n \geq n_{\varepsilon}$. Observe that $\left\langle g_{i}\left(\cdot, v^{m}\right)-g_{i}(\cdot, v),. \varphi_{n_{\varepsilon}}\right\rangle \rightarrow 0$, so it is possible to choose $m_{\varepsilon}$ to conclude that the claim is true.

(iii) Consider the following condition: 
(IV') there exists $C_{g}>0$ such that $\forall t \in[0, T], \forall u, v \in C^{0}([-h, T] ; V)$ and for every bounded subset $\mathcal{O} \subset \Omega$

$$
\int_{0}^{t}\left\|g\left(s, u_{s}\right)-g\left(s, v_{s}\right)\right\|_{\left(L^{2}(\mathcal{O})\right)^{N}}^{2} \mathrm{~d} s \leq C_{g} \int_{-h}^{t}\|u(s)-v(s)\|_{\left(L^{2}(\mathcal{O})\right)^{N}}^{2} \mathrm{~d} s,
$$

where the norm $\|\cdot\|_{\left(L^{2}(\mathcal{O})\right)^{N}}$ is understood as the restriction to $\mathcal{O}$ of the concerned functions.

Observe that if $g_{1}$ satisfies (I)-(IV) and (IV'), then, as a direct consequence of (IV'), $g_{1}$ satisfies assumption (V).

\section{Some general situations}

In this section, we are going to show some situations where our theory can be applied. The cases considered include situations of variable and distributed delay. For other examples, see [Caraballo \& Real 2003].

\section{(a) Case 1}

Let $G:[0, T] \times \mathbb{R}^{N} \rightarrow \mathbb{R}^{N}$ be a measurable function satisfying $G(t, 0)=0$ for all $t \in[0, T]$, and assume that there exists $L_{1}>0$ such that

$$
|G(t, u)-G(t, v)|_{\mathbb{R}^{N}} \leq L_{1}|u-v|_{\mathbb{R}^{N}}, \forall u, v \in \mathbb{R}^{N} .
$$

Consider a function $\rho(t)$, which is going to play the role of the delay function. We suppose that $\rho \in C^{1}([0, T]), \rho(t) \geq 0$ for all $t \in[0, T], h=\max _{t \in[0, T]} \rho(t)>0$ and $\rho_{*}=\max _{t \in[0, T]} \rho^{\prime}(t)<1$. Then, we define $g_{1}(t, \xi)(x)=G(t, \xi(-\rho(t))(x))$ for each $\xi \in C^{0}([-h, 0] ; H), x \in \Omega$ and $t \in[0, T]$. Notice that, in this case, the delayed term $g_{1}$ in our problem turns into $g_{1}\left(t, u_{t}\right)=G(t, u(t-\rho(t)))$. Then, $g_{1}$ satisfies the corresponding hypotheses in Theorem 2.3.

Indeed, (I)-(III) follow immediately. On the other hand, if $u, v \in L^{2}(-h, T ; H)$, using the change of variable $\tau=s-\rho(s)$ it is easy to see that

$$
\int_{0}^{t}\left\|g_{1}\left(s, u_{s}\right)-g_{1}\left(s, v_{s}\right)\right\|_{\left(L^{2}(\mathcal{O})\right)^{N}}^{2} \mathrm{~d} s \leq \int_{-h}^{t}\|u(\tau)-v(\tau)\|_{\left(L^{2}(\mathcal{O})\right)^{N}}^{2} \mathrm{~d} \tau \quad \forall t \in[0, T],
$$

for any $\mathcal{O} \subset \Omega$ and, consequently, (IV), (IV') and (V) are fulfilled.

\section{(b) Case 2}

Let now $G:[0, T] \times[-h, 0] \times \mathbb{R}^{N} \rightarrow \mathbb{R}^{N}$ be a measurable function satisfying $G(t, s, 0)=0$ for all $(t, s) \in[0, T] \times[-h, 0]$ and such that there exists a function $\gamma \in L^{2}(-h, 0)$ such that

$$
|G(t, s, u)-G(t, s, v)|_{\mathbb{R}^{N}} \leq \gamma(s)|u-v|_{\mathbb{R}^{N}}, \forall u, v \in \mathbb{R}^{N} \quad \forall(t, s) \in[0, T] \times[-h, 0] .
$$

Then, we define $g_{1}(t, \xi)(x)=\int_{-h}^{0} G(t, s, \xi(s)(x)) \mathrm{d} s$ for each $\xi \in C^{0}([0, T] ; H)$, $x \in \Omega$ and $t \in[0, T]$. In this case, the delayed term $g_{1}$ in our problem becomes

$$
g_{1}\left(t, u_{t}\right)=\int_{-h}^{0} G(t, s, u(t+s)) \mathrm{d} s
$$


As in Case 1, $g_{1}$ satisfies the hypotheses in Theorem 2.3 with $X=H$ and $Y=$ $\left(L^{2}(\Omega)\right)^{N}$.

Indeed, (I) and (II) can be deduced immediately. On the other hand, if $\xi, \eta \in$ $C^{0}([0, T] ; H)$, for each $t \in[0, T]$ we obtain

$$
\begin{aligned}
\left|g_{1}(t, \xi)-g_{1}(t, \eta)\right|^{2} & \leq \int_{\Omega}\left(\int_{-h}^{0}|G(t, s, \xi(s)(x))-G(t, s, \eta(s)(x))|_{\mathbb{R}^{N}} \mathrm{~d} s\right)^{2} \mathrm{~d} x \\
& \leq \int_{\Omega}\left(\int_{-h}^{0} \gamma(s)|\xi(s)(x)-\eta(s)(x)|_{\mathbb{R}^{N}} \mathrm{~d} s\right)^{2} \mathrm{~d} x \\
& \leq \int_{\Omega}\|\gamma\|_{L^{2}(-h, 0)}^{2}\left(\int_{-h}^{0}|\xi(s)(x)-\eta(s)(x)|_{\mathbb{R}^{N}}^{2} \mathrm{~d} s\right) \mathrm{d} x \\
& \leq h\|\gamma\|_{L^{2}(-h, 0)}^{2}\|\xi-\eta\|_{C^{0}([0, T] ; H)}^{2} .
\end{aligned}
$$

Finally, if $u, v \in L^{2}(-h, T ; H)$ then, for each $t \in[0, T]$ it follows

$\int_{0}^{t}\left|g_{1}\left(\tau, u_{\tau}\right)-g_{1}\left(\tau, v_{\tau}\right)\right|^{2} \mathrm{~d} \tau \leq h\|\gamma\|_{L^{2}(-h, 0)}^{2} \int_{0}^{t}\left(\int_{-h}^{0}|u(s+\tau)-v(s+\tau)|^{2} \mathrm{~d} s\right) \mathrm{d} \tau$, and, with the change $r=s+\tau$,

$$
\begin{aligned}
\int_{0}^{t}\left|g_{1}\left(\tau, u_{\tau}\right)-g_{1}\left(\tau, v_{\tau}\right)\right|^{2} \mathrm{~d} \tau & \leq h\|\gamma\|_{L^{2}(-h, 0)}^{2} \int_{0}^{t}\left(\int_{\tau-h}^{\tau}|u(r)-v(r)|^{2} \mathrm{~d} r\right) \mathrm{d} \tau \\
& \leq h T\|\gamma\|_{L^{2}(-h, 0)}^{2} \int_{-h}^{t}|u(r)-v(r)|^{2} \mathrm{~d} r .
\end{aligned}
$$

Condition (V) can be checked similarly.

\section{Stability of stationary solutions}

In this section, we prove existence and uniqueness of stationary solutions to our Navier-Stokes model for dimensions $N=2$ or 3 , when the delay term has a special form, provided the viscosity is large enough. Additionally, for $N=2$, we see that all the solutions to the evolutionary problem converge to the (unique) stationary solution exponentially fast.

From here on we suppose $f$ is independent of time, and the delay term has the form $g\left(t, u_{t}\right)=G(u(t-\rho(t))$ with $G$ as in Case 1 but independent of time too, i.e. $G: \mathbb{R}^{N} \rightarrow \mathbb{R}^{N}$ satisfies $G(0)=0$ and there exists $L_{1}>0$ such that

$$
|G(u)-G(v)|_{\mathbb{R}^{N}} \leq L_{1}|u-v|_{\mathbb{R}^{N}}, \forall u, v \in \mathbb{R}^{N} .
$$

So we will consider the problem

$$
\left\{\begin{array}{l}
\text { To find } u \in L^{2}(-h, T ; V) \cap L^{\infty}(0, T ; H) \text { such that, for all } v \in V, \\
\frac{\mathrm{d}}{\mathrm{d} t}(u(t), v)+\nu a(u(t), v)+b(u(t), u(t), v)=\langle f, v\rangle+\left(G\left(u_{t}\right), v\right), \\
u(0)=u^{0}, u(t)=\phi(t), \quad t \in(-h, 0) .
\end{array}\right.
$$

A stationary solution to (4.1) is a function $u^{*} \in V$ such that

$$
\nu a\left(u^{*}, v\right)+b\left(u^{*}, u^{*}, v\right)=\langle f, v\rangle+\left(G\left(u^{*}\right), v\right) \quad \text { for all } v \in V .
$$

Then, we may establish the following 
Theorem 4.1. Suppose that $G$ satisfies the conditions above and $\nu>\lambda_{1}^{-1} L_{1}$. Then,

(a) For all $f \in V^{\prime}$ there exists at least a solution to (4.2);

(b) The following conditions are sufficient to have uniqueness of solution to $(4.2)$ :

$$
\left(\nu-\lambda_{1}^{-1} L_{1}\right)^{2}>\left(2 \lambda_{1}\right)^{-1 / 2}\|f\|_{*} \quad \text { for } N=2,
$$

and

$$
\left(\nu-\lambda_{1}^{-1} L_{1}\right)^{2}>2^{-1} \lambda_{1}^{-1 / 4}\|f\|_{*} \quad \text { for } N=3 .
$$

Proof. (a) As before, we consider $B=\left\{w_{1}, w_{2}, \ldots\right\}$ an ortonormal basis in $H$ composed by elements of $\mathcal{V}$ such that their linear combinations are dense in $V$.

Since compact injection is not valid in this context, we modify the arguments in Simon 2003 and in Caraballo \& Real 2003 (Theorem 3.1). We will proceed in three steps, by a Galerkin argument and using Brouwer fixed point theorem on each projected equation.

Step 1: Denote $V_{m}=\operatorname{span}\left[w_{1}, \ldots, w_{m}\right]$ and consider, for a fixed $z^{m} \in V_{m}$, the problem of finding $u^{m} \in V_{m}$ such that solves the equation

$$
\nu\left(\left(u^{m}, v^{m}\right)\right)+b\left(z^{m}, u^{m}, v^{m}\right)=\left\langle f, v^{m}\right\rangle+\left(G\left(z^{m}\right), v^{m}\right) \forall v^{m} \in V_{m}
$$

Observe that for each $z^{m} \in V_{m}$ the functional $(u, v) \mapsto \nu((u, v))+b\left(z^{m}, u, v\right)$ is bilinear, continuous and coercive in $V_{m} \times V_{m}\left(V_{m}\right.$ is considered with the norm $\|\cdot\|$ of $V$ ). On other hand, the functional in $V_{m}$ defined by $v \mapsto\langle f, v\rangle+\left(G\left(z^{m}\right), v\right)$ is obviously linear and continuous. So, by the Lax-Milgram Theorem, for each fixed $z^{m} \in V_{m}$, there exists a unique solution to (4.3), which we denote $u^{m}$. Define $T_{m}: V_{m} \rightarrow V_{m}$ the operator given by $T_{m}\left(z^{m}\right)=u^{m}$.

Step 2: We will see that for each $m$ we may apply a fixed point theorem to the map $T_{m}$ (restricted to a suitable subset $K_{m} \subset V_{m}$ ) to ensure that we can obtain existence of $u^{m} \in V_{m}$ such that

$$
\nu\left(\left(u^{m}, v^{m}\right)\right)+b\left(u^{m}, u^{m}, v^{m}\right)=\left\langle f, v^{m}\right\rangle+\left(G\left(u^{m}\right), v^{m}\right) \forall v^{m} \in V_{m}
$$

In order to proceed, we take in $(4.3) v^{m}=u^{m}$, which leads by standard estimations to the following

$$
\nu\left\|u^{m}\right\|^{2} \leq\|f\|_{*}\left\|u^{m}\right\|+\lambda_{1}^{-1} L_{1}\left\|z^{m}\right\|\left\|u^{m}\right\| \Rightarrow \nu\left\|u^{m}\right\| \leq\|f\|_{*}+\lambda_{1}^{-1} L_{1}\left\|z^{m}\right\| .
$$

As long as we assume $\nu>\lambda_{1}^{-1} L_{1}$, one may take $k>0$ such that $k\left(\nu-\lambda_{1}^{-1} L_{1}\right) \geq$ $\|f\|_{*}$, so we conclude $\nu\left\|u^{m}\right\| \leq k \nu-k \lambda_{1}^{-1} L_{1}+\lambda_{1}^{-1} L_{1}\left\|z^{m}\right\|$. We define now $K_{m}=$ $\left\{z \in V_{m}:\|z\| \leq k\right\}$, which is a convex set of $V$, and indeed compact. Observe that the application $T_{m}$ maps $K_{m}$ into itself.

We will complete this step applying the Brouwer fixed point theorem to $\left.T_{m}\right|_{K_{m}}$. For this goal, it only remains to check that $T_{m}$ is continuous. Indeed, take $z_{1}^{m}$ and $z_{2}^{m} \in V_{m}$ and denote by $u_{i}^{m}=T\left(z_{i}^{m}\right)$ the respective solutions to equations (4.4). So, taking the difference

$$
\nu\left(\left(u_{1}^{m}-u_{2}^{m}, v^{m}\right)\right)+b\left(z_{1}^{m}, u_{1}^{m}, v^{m}\right)-b\left(z_{2}^{m}, u_{2}^{m}, v^{m}\right)=\left(G\left(z_{1}^{m}\right)-G\left(z_{2}^{m}\right), v^{m}\right)
$$


for all $v^{m} \in V_{m}$. In particular, if we put $v^{m}=u_{1}^{m}-u_{2}^{m}$, and since $b(u, v, v)=0$, we have the required continuity of $\left.T_{m}\right|_{K_{m}}$ :

$$
\begin{aligned}
\nu\left\|u_{1}^{m}-u_{2}^{m}\right\|^{2}= & b\left(z_{2}^{m}, u_{2}^{m}, u_{1}^{m}-u_{2}^{m}\right)-b\left(z_{1}^{m}, u_{1}^{m}, u_{1}^{m}-u_{2}^{m}\right) \\
& +\left(G\left(z_{1}^{m}\right)-G\left(z_{2}^{m}\right), u_{1}^{m}-u_{2}^{m}\right) \\
= & b\left(z_{2}^{m}, u_{2}^{m}, u_{1}^{m}-u_{2}^{m}\right) \pm b\left(z_{2}^{m}, u_{1}^{m}, u_{1}^{m}-u_{2}^{m}\right)-b\left(z_{1}^{m}, u_{1}^{m}, u_{1}^{m}-u_{2}^{m}\right) \\
& +\left(G\left(z_{1}^{m}\right)-G\left(z_{2}^{m}\right), u_{1}^{m}-u_{2}^{m}\right) \\
= & b\left(z_{2}^{m}-z_{1}^{m}, u_{1}^{m}, u_{1}^{m}-u_{2}^{m}\right)+\left(G\left(z_{1}^{m}\right)-G\left(z_{2}^{m}\right), u_{1}^{m}-u_{2}^{m}\right) .
\end{aligned}
$$

Taking into account that $u_{1}^{m} \in K_{m}$, this can be bounded by

$$
\nu\left\|u_{1}^{m}-u_{2}^{m}\right\|^{2} \leq \begin{cases}\left(\left(2 \lambda_{1}\right)^{-1 / 2} k+\lambda_{1}^{-1} L_{1}\right)\left\|z_{2}^{m}-z_{1}^{m}\right\|\left\|u_{1}^{m}-u_{2}^{m}\right\| & \text { if } N=2, \\ \left(2^{-1} \lambda_{1}^{-1 / 4} k+\lambda_{1}^{-1} L_{1}\right)\left\|z_{2}^{m}-z_{1}^{m}\right\|\left\|u_{1}^{m}-u_{2}^{m}\right\| & \text { if } N=3 .\end{cases}
$$

Step 3: We will pass to the limit on the solutions obtained in Step 2 to conclude the existence of a solution of (4.2).

Put $v^{m}=u^{m}$ in (4.4), so

$$
\nu\left\|u^{m}\right\|^{2}=\left\langle f, u^{m}\right\rangle+\left(G\left(u^{m}\right), u^{m}\right) \leq\|f\|_{*}\left\|u^{m}\right\|+\lambda_{1}^{-1} L_{1}\left\|u^{m}\right\|^{2} .
$$

This gives a uniform bound in $V$ for all the solutions obtained in Step 2: $\left\|u^{m}\right\| \leq$ $\|f\|_{*} /\left(\nu-\lambda_{1}^{-1} L_{1}\right)$. [Actually this is redundant since we did check this as the optimal value $k$ in Step 2, valid bound for all the fixed point with the norm $\|\cdot\|$ on every $K_{m}$.] We may extract a weakly convergent subsequence (which we relabel the same): $u^{m} \rightarrow u$ in $V$. Moreover, for any regular bounded set $\mathcal{O} \subset \Omega$, we have the same uniform bounds of $\left.u^{m}\right|_{\mathcal{O}}$, which means, using now the compact injection, that $\left.\left.u^{m}\right|_{\mathcal{O}} \rightarrow u\right|_{\mathcal{O}}$ in $\left(L^{2}(\mathcal{O})\right)^{N}$.

To proceed, we fix any $w_{j} \in B$. Since we have a (sub-)sequence of equations (4.4) for every $m$ greater than $j$, it is clear we may pass through the limit on every term to obtain

$$
\nu\left(\left(u, w_{j}\right)\right)+b\left(u, u, w_{j}\right)=\left\langle f, w_{j}\right\rangle+\left(G(u), w_{j}\right) .
$$

The first term is obtained by the weak convergence in $V u^{m} \rightarrow u$. The trilinear terms converge as long as they have sense on the support of $w_{j}$ which is compact (denote by $\mathcal{O}_{j} \subset \Omega$ a bounded open set with smooth boundary containing it); thus we not only have the weak convergence $u^{m} \rightarrow u$ in $V$ but the strong convergence $u^{m} \rightarrow u$ in $\left(L^{2}\left(\mathcal{O}_{j}\right)\right)^{N}$. Finally, for the terms $\left(G\left(u^{m}\right), w_{j}\right)$ we have something similar:

$$
\left|\left(G\left(u^{m}\right), w_{j}\right)-\left(G(u), w_{j}\right)\right| \leq\left\|G\left(u^{m}\right)-G(u)\right\|_{\left(L^{2}\left(\mathcal{O}_{j}\right)\right)^{N}}\left|w_{j}\right| \leq L_{1}\left\|u^{m}-u\right\|_{\left(L^{2}\left(\mathcal{O}_{j}\right)\right)^{N}}\left|w_{j}\right|
$$

which goes to zero by the strong convergence in $\left(L^{2}\left(\mathcal{O}_{j}\right)\right)^{N}$.

Thus, we deduce (4.5) holds for each $w_{j}$. Since the linear combinations of elements of $B=\left\{w_{1}, w_{2}, \ldots\right\}$ is dense in $V$, we conclude that (4.2) is satisfied at least by $u^{*}=u$.

(b) Now we prove uniqueness of solution to (4.2) under the suitable extra assumptions for $N=2$ and 3 . 
Firstly, as we will use it below, we give an estimate on the solution we know there exists. Putting $v=u^{*}$ in (4.2), this gives $\left\|u^{*}\right\| \leq\|f\|_{*} /\left(\nu-\lambda_{1}^{-1} L_{1}\right)$. Actually, every solution to (4.2) must satisfy this bound.

Suppose there are two solutions $u_{1}$ and $u_{2}$ to (4.2). Then,

$$
\nu\left(\left(u_{1}-u_{2}, v\right)\right)+b\left(u_{1}, u_{1}, v\right)-b\left(u_{2}, u_{2}, v\right)=\left(G\left(u_{1}\right)-G\left(u_{2}\right), v\right) \text { for all } v \in V .
$$

Taking $v=u_{1}-u_{2}$ and using, as before, that $b(u, v, v)=0$, this leads (for $N=2$ ) to

$$
\begin{aligned}
\nu\left\|u_{1}-u_{2}\right\|^{2} & =b\left(u_{2}-u_{1}, u_{1}, u_{1}-u_{2}\right)+\left(G\left(u_{1}\right)-G\left(u_{2}\right), u_{1}-u_{2}\right) \\
& \leq 2^{-1 / 2}\left|u_{2}-u_{1}\right|\left\|u_{1}\right\|\left\|u_{2}-u_{1}\right\|+\lambda_{1}^{-1} L_{1}\left\|u_{2}-u_{1}\right\|^{2}
\end{aligned}
$$

Using the above estimation over any solution $\left\|u_{1}\right\|$, and the Poincaré condition (1.1), we conclude that

$$
\nu\left\|u_{1}-u_{2}\right\|^{2} \leq \frac{\left(2 \lambda_{1}\right)^{-1 / 2}\|f\|_{*}}{\nu-\lambda_{1}^{-1} L_{1}}\left\|u_{2}-u_{1}\right\|^{2}+\lambda_{1}^{-1} L_{1}\left\|u_{2}-u_{1}\right\|^{2},
$$

whence

$$
\left(\nu-\lambda_{1}^{-1} L_{1}\right)^{2}\left\|u_{1}-u_{2}\right\|^{2} \leq\left(2 \lambda_{1}\right)^{-1 / 2}\|f\|_{*}\left\|u_{2}-u_{1}\right\|^{2},
$$

and the uniqueness follows for $N=2$ as long as we suppose $\left(\nu-\lambda_{1}^{-1} L_{1}\right)^{2}>$ $\left(2 \lambda_{1}\right)^{-1 / 2}\|f\|_{*}$.

The case $N=3$ follows analogously:

$$
\begin{aligned}
\left|b\left(u_{2}-u_{1}, u_{1}, u_{1}-u_{2}\right)\right| & \leq 2^{-1}\left\|u_{1}\right\|\left|u_{2}-u_{1}\right|^{1 / 2}\left\|u_{2}-u_{1}\right\|^{3 / 2} \\
& \leq 2^{-1} \lambda_{1}^{-1 / 4} \frac{\|f\|_{*}}{\nu-\lambda_{1}^{-1} L_{1}}\left\|u_{2}-u_{1}\right\|^{2}
\end{aligned}
$$

whence

$$
\left(\nu-\lambda_{1}^{-1} L_{1}\right)^{2}\left\|u_{1}-u_{2}\right\|^{2} \leq 2^{-1} \lambda_{1}^{-1 / 4}\|f\|_{*}\left\|u_{1}-u_{2}\right\|^{2} .
$$

The next result is stated for $N=2$ and gives another condition, slightly stronger than (a) in Theorem 4.1, and therefore ensuring again existence and uniqueness of stationary solution of (4.2), denoted $u^{*}$, but such that every weak solution of the evolutionary problem (1.2) approaches $u^{*}$ exponentially fast as $t$ goes to $+\infty$. This result is an improvement of Theorem 3.3 in Caraballo \& Real 2003, both in the condition (4.6) below and the allowed force field $f$.

Theorem 4.2. Consider $G: \mathbb{R}^{2} \rightarrow \mathbb{R}^{2}$ a Lipschitz map satisfying $G(0)=0$, and with Lipschitz constant $L_{1}>0$. Assume that the forcing term $g\left(t, u_{t}\right)$ in (1.2) is given by $g\left(t, u_{t}\right)=G\left(u(t-\rho(t))\right.$ with $\rho \in C^{1}\left(\mathbb{R}_{+} ;[0, h]\right)$ such that $\rho^{\prime}(t) \leq \rho_{*}<1$ for all $t \geq 0$. Suppose also that $f \in V^{\prime}, \nu>\lambda_{1}^{-1} L_{1}$, and the following inequality is satisfied in addition:

$$
\nu \lambda_{1}>\frac{L_{1}}{\left(1-\rho_{*}\right)^{1 / 2}}+\frac{\left(2^{-1} \lambda_{1}\right)^{1 / 2}\|f\|_{*}}{\nu-\lambda_{1}^{-1} L_{1}} .
$$


Then there is a unique stationary solution $u^{*}$ of (4.2) and every solution of (1.2) converges to $u^{*}$ exponentially fast as $t \rightarrow+\infty$, that is, there exist two positive constants $C$ and $\lambda$, such that for all $u^{0} \in H$ and $\phi \in L^{2}(-h, 0 ; V)$, the solution $u$ of (1.2) with $f(t) \equiv f$ satisfies for all $t \geq 0$ :

$$
\left|u(t)-u^{*}\right|^{2} \leq C e^{-\lambda t}\left(\left|u^{0}-u^{*}\right|^{2}+\left\|\phi-u^{*}\right\|_{L^{2}(-h, 0 ; V)}^{2}\right) .
$$

Proof. Consider $u$ the solution of (1.2) for $f(t) \equiv f$, and denote $u^{*} \in V$ the stationary solution to (4.2), which existence and uniqueness is ensured by Theorem 4.1. Set $w(t)=u(t)-u^{*}$, and observe that

$\frac{\mathrm{d}}{\mathrm{d} t}(w(t), v)+\nu((w(t), v))+b(u(t), u(t), v)-b\left(u^{*}, u^{*}, v\right)=(G(u(t-\rho(t))), v)-\left(G\left(u^{*}\right), v\right)$.

Since

$$
\begin{aligned}
& b(u(t), u(t), w(t))-b\left(u^{*}, u^{*}, w(t)\right) \\
= & b\left(u^{*}, w(t), u^{*}\right)-b(u(t), w(t), u(t)) \\
= & b\left(u^{*}, w(t), u^{*}\right) \mp b\left(u(t), w(t), u^{*}\right)-b(u(t), w(t), u(t)) \\
= & -b\left(w(t), w(t), u^{*}\right)-b(u(t), w(t), w(t)) \\
= & -b\left(w(t), w(t), u^{*}\right),
\end{aligned}
$$

we can obtain the following estimation (here $\lambda$ and $\delta$ are fixed positive values to be determined later on):

$$
\begin{aligned}
\frac{\mathrm{d}}{\mathrm{d} t}\left(\mathrm{e}^{\lambda t}|w(t)|^{2}\right)= & \lambda \mathrm{e}^{\lambda t}|w(t)|^{2}+\mathrm{e}^{\lambda t} \frac{\mathrm{d}}{\mathrm{d} t}|w(t)|^{2} \\
= & \lambda \mathrm{e}^{\lambda t}|w(t)|^{2}+2 \mathrm{e}^{\lambda t}\left(-\nu\|w(t)\|^{2}-b(u(t), u(t), w(t))\right. \\
& \left.+b\left(u^{*}, u^{*}, w(t)\right)+\left(G(u(t-\rho(t)))-G\left(u^{*}\right), w(t)\right)\right) \\
\leq & \mathrm{e}^{\lambda t}\left(\lambda|w(t)|^{2}-2 \nu\|w(t)\|^{2}+2 b\left(w(t), w(t), u^{*}\right)\right. \\
& \left.+2 L_{1}|w(t-\rho(t)) \| w(t)|\right) \\
\leq & \lambda_{1}^{-1} \mathrm{e}^{\lambda t}\left(\lambda+\delta L_{1}-2 \nu \lambda_{1}\right)\|w(t)\|^{2}+2 \mathrm{e}^{\lambda t}\left|b\left(w(t), w(t), u^{*}\right)\right| \\
& +\left(\delta \lambda_{1}\right)^{-1} L_{1} \mathrm{e}^{\lambda t}\|w(t-\rho(t))\|^{2}
\end{aligned}
$$

Using again that $\left|b\left(w(t), w(t), u^{*}\right)\right| \leq\left(2 \lambda_{1}\right)^{-1 / 2}\|w(t)\|^{2}\left\|u^{*}\right\|$ and taking into account the estimate we proved in Step 3 of Theorem 4.1 for the stationary solution, $\left\|u^{*}\right\| \leq$ $\|f\|_{*} /\left(\nu-\lambda_{1}^{-1} L_{1}\right)$, we have

$$
\left|b\left(w(t), w(t), u^{*}\right)\right| \leq \frac{\left(2 \lambda_{1}\right)^{-1 / 2}\|f\|_{*}}{\nu-\lambda_{1}^{-1} L_{1}}\|w(t)\|^{2} .
$$

Substituting this last inequality into (4.8) it follows that

$$
\begin{aligned}
\frac{\mathrm{d}}{\mathrm{d} t}\left(\mathrm{e}^{\lambda t}|w(t)|^{2}\right) \leq & \lambda_{1}^{-1} \mathrm{e}^{\lambda t}\left(\lambda+\delta L_{1}-2 \nu \lambda_{1}+\frac{\left(2 \lambda_{1}\right)^{1 / 2}\|f\|_{*}}{\nu-\lambda_{1}^{-1} L_{1}}\right)\|w(t)\|^{2} \\
& +\left(\delta \lambda_{1}\right)^{-1} L_{1} \mathrm{e}^{\lambda t}\|w(t-\rho(t))\|^{2}
\end{aligned}
$$

Article submitted to Nonlinear Analysis TMA 
and so for all $t \in[0, T]$

$$
\begin{aligned}
\mathrm{e}^{\lambda t}|w(t)|^{2} \leq & |w(0)|^{2}+\left(\delta \lambda_{1}\right)^{-1} L_{1} \int_{0}^{t} \mathrm{e}^{\lambda s}\|w(s-\rho(s))\|^{2} \mathrm{~d} s \\
& +\lambda_{1}^{-1}\left(\lambda+\delta L_{1}-2 \nu \lambda_{1}+\frac{\left(2 \lambda_{1}\right)^{1 / 2}\|f\|_{*}}{\nu-\lambda_{1}^{-1} L_{1}}\right) \int_{0}^{t} \mathrm{e}^{\lambda s}\|w(s)\|^{2} \mathrm{~d} s .
\end{aligned}
$$

We concentrate momentarily in the delay term on the right hand side. Observing that the function $\phi(t):=t-\rho(t)$ is strictly increasing, that $\rho$ takes values on $[0, h]$, and so $\phi^{-1}(\eta) \leq \eta+h$, we can apply the change of variable $\eta=s-\rho(s)=\phi(s)$ :

$$
\begin{aligned}
\int_{0}^{t} \mathrm{e}^{\lambda s}\|w(s-\rho(s))\|^{2} \mathrm{~d} s & =\int_{-\rho(0)}^{t-\rho(t)} \mathrm{e}^{\lambda \tau^{-1}(\eta)}\|w(\eta)\|^{2} \frac{1}{1-\rho^{\prime}\left(\phi^{-1}(\eta)\right)} \mathrm{d} \eta \\
& \leq \frac{\mathrm{e}^{\lambda h}}{1-\rho_{*}} \int_{-h}^{t} \mathrm{e}^{\lambda \eta}\|w(\eta)\|^{2} \mathrm{~d} \eta .
\end{aligned}
$$

Combining the above two inequalities we obtain

$$
\begin{aligned}
\mathrm{e}^{\lambda t}|w(t)|^{2} \leq & |w(0)|^{2}+\left(\delta \lambda_{1}\right)^{-1} L_{1} \frac{\mathrm{e}^{\lambda h}}{1-\rho_{*}} \int_{-h}^{t} \mathrm{e}^{\lambda s}\|w(s)\|^{2} \mathrm{~d} s \\
& +\lambda_{1}^{-1}\left(\lambda+\delta L_{1}-2 \nu \lambda_{1}+\frac{\left(2 \lambda_{1}\right)^{1 / 2}\|f\|_{*}}{\nu-\lambda_{1}^{-1} L_{1}}\right) \int_{0}^{t} \mathrm{e}^{\lambda s}\|w(s)\|^{2} \mathrm{~d} s .
\end{aligned}
$$

Observe the coefficients of the integral $\int_{0}^{t} \mathrm{e}^{\lambda s}\|w(s)\|^{2} \mathrm{~d} s$. Let us note that $\delta_{*}=$ $\left(1-\rho_{*}\right)^{-1 / 2}$ is the minimum of the map $\delta \mapsto \delta+1 /\left(\delta\left(1-\rho_{*}\right)\right)$. Then, thanks to (4.6) there exists $\lambda>0$ small enough such that

$$
\lambda+\delta_{*} L_{1}-2 \nu \lambda_{1}+\frac{\left(2 \lambda_{1}\right)^{1 / 2}\|f\|_{*}}{\nu-\lambda_{1}^{-1} L_{1}}+\frac{L_{1} \mathrm{e}^{\lambda h}}{\delta_{*}\left(1-\rho_{*}\right)} \leq 0 .
$$

Thus, we deduce that

$$
\mathrm{e}^{\lambda t}\left|u(t)-u^{*}\right|^{2} \leq\left|u^{0}-u^{*}\right|^{2}+\frac{\lambda_{1}^{-1} L_{1} \mathrm{e}^{\lambda h}}{1-\rho_{*}} \int_{-h}^{0} \mathrm{e}^{\lambda \eta}\|w(\eta)\|^{2} \mathrm{~d} \eta,
$$

whence (4.7) is satisfied with $C=\max \left\{1, \frac{\lambda_{1}^{-1} L_{1} \mathrm{e}^{\lambda h}}{1-\rho_{*}}\right\}$.

Remark 4.3. The above result has been given with $g_{1}$ as in Case 1 of Section 3 to simplify the notation. Other types of delay could be used, for example one could consider an autonomous case with distributed delay by removing in Case 2 the dependence of $G$ on its first variable.

\section{Conclusions and final comments}

Existence, uniqueness and stability results have been established under different conditions - essentially viscosity is asked to be large enough-. One may wonder about results under weaker assumptions, where uniqueness or stability may not be 
ensured. This leads us to consider additional concepts from the theory of dynamical systems, namely attractors, both the classical (forward) one, and the 'pullback' definition that is well-suited to non-autonomous systems (see Caraballo \& Real 2004 and Kloeden \& Schmalfuß 1997).

\section{Acknowledgement}

This work was partially supported by Ministerio de Ciencia y Tecnología (Spain) and FEDER Proyecto BFM2002-03068. The authors thanks Professor José Real for his advice on the subject of this paper.

\section{References}

Artola, M. 1969 Sur les perturbations des équations d'evolution, application à des problèmes de retard, Ann. Scient. Ec. Norm. Sup. 4 série, t. 2, 137-253.

Bensoussan, A., Da Prato, G., Delfour, M.C., \& Mitter, S.K. 1992 Representation and Control of Infinite Dimensional Systems, Vol. I, Birkhäuser, Boston-Basel-Berlin.

Caraballo, T. \& Real, J. 2001 Navier-Stokes equations with delays, Proc. R. Soc. Lond. A(2001) 457, 2441-2453.

Caraballo, T. \& Real, J. 2003 Asymptotic behaviour of $2 D-$ Navier-Stokes equations with delays, Proc. R. Soc. Lond. A(2003) 459, 3181-3194.

Caraballo, T. \& Real, J. 2004 Attractors for 2D-Navier-Stokes models with delays, J. Differential Equations 205, 271-297.

Constantin, P. \& Foias, C. 1988 Navier Stokes Equations, The University of Chicago Press, Chicago.

Hale, J.K. \& Verduyn Lunel, S.M. 1995 Introduction to Functional Differential Equations, Springer-Verlag, New York.

Kloeden, P. E. \& Schmalfuß, B. 1997 Nonautonomous systems, cocycle attractors and variable time-step discretization. Dynamical numerical analysis (Atlanta, GA, 1995). Numer. Algorithms 14, 141-152.

Ladyzhenskaya, O. 1992 First boundary value problem for the Navier-Stokes equations in domains with nonsmooth boundaries. C. R. Acad. Sci. Paris Sér. I Math. 314, 253-258.

Leray, J. 1933 Etude de diverses équations intégrales non linéaires et de quelques problèmes que pose l'hydrodynamique, J. Math. Pures Appl. 12, 1-82.

Lions, J.L. 1969 Quelques méthodes de résolution des problèmes aux limites non linéaires, Dunod, Gauthier- Villars, Paris.

Rosa, R. 1998 The global attractor for the 2D Navier-Stokes flow on some unbounded domains., Nonlinear Anal. 32, 71-85.

Simon, J. 2003 Équations de Navier-Stokes, Cours de DEA 2002-2003, Université Blaise Pascal. http://wwwlma.univ-bpclermont.fr/ simon/

Temam, R. 1979 Navier-Stokes equations, Theory and Numerical Analysis, 2nd. ed., North Holland, Amsterdam. 